Claremont Colleges

Scholarship@ Claremont

Pomona Faculty Publications and Research

Pomona Faculty Scholarship

$12-6-2006$

\title{
Modified Control Software for Imaging Ultracold Atomic Clouds
}

Dwight L. Whitaker

Pomona College

A. Sharma

J.M. Brown

\section{Recommended Citation}

"Modified control software for imaging ultracold atomic clouds", D. L. Whitaker, A. Sharma, and J. M. Brown, Rev. Sci. Instr., 77, 126101 (2006). DOI: 10.1063/1.2400012.

This Article is brought to you for free and open access by the Pomona Faculty Scholarship at Scholarship @ Claremont. It has been accepted for inclusion in Pomona Faculty Publications and Research by an authorized administrator of Scholarship @ Claremont. For more information, please contact scholarship@cuc.claremont.edu. 


\title{
Modified control software for imaging ultracold atomic clouds
}

\author{
D. L. Whitaker, ${ }^{\text {a) }}$ A. Sharma, and J. M. Brown ${ }^{\text {b) }}$ \\ Physics Department, Williams College, Williamstown, Massachusetts 01267
}

(Received 15 September 2006; accepted 23 October 2006; published online 6 December 2006)

\begin{abstract}
A charge-coupled device (CCD) camera capable of taking high-quality images of ultracold atomic samples can often represent a significant portion of the equipment costs in atom trapping experiment. We have modified the commercial control software of a CCD camera designed for astronomical imaging to take absorption images of ultracold rubidium clouds. This camera is sensitive at $780 \mathrm{~nm}$ and has been modified to take three successive 16-bit images at full resolution. The control software can be integrated into a Matlab graphical user interface with fitting routines written as Matlab functions. This camera is capable of recording high-quality images at a fraction of the cost of similar cameras typically used in atom trapping experiments. (C) 2006 American Institute of Physics. [DOI: 10.1063/1.2400012]
\end{abstract}

\section{INTRODUCTION}

A number of technical advances have facilitated experiments to produce samples of ultracold atoms. Magnetooptical traps (MOT) can be made using simple, inexpensive, external cavity diode lasers ${ }^{1-3}$ at a cost suitable for undergraduate laboratories. ${ }^{4}$ Atoms precooled in a MOT can be cooled to quantum degeneracy in a variety of novel, simple traps suited for a number of applications. A movable quadrupole magnetic trap ${ }^{5}$ can transfer atoms to a magnetic trap in an UHV chamber with excellent optical access. One can also create ultracold atoms without exceptional vacuum in the extremely tight traps produced from the magnetic field of wires on a chip ${ }^{6}$ or from a focused off-resonant laser beam. $^{7-9}$

Despite the technological improvements that have made creating Bose-Einstein condensates (BECs) and ultracold atomic samples easier and cheaper, the technology of taking measurements with a charge-coupled device (CCD) image has received less attention. Essentially all data on trapped clouds are taken either by measuring the absorption (absorption imaging) ${ }^{10}$ or by recording the phase shift (phase contrast imaging) ${ }^{11}$ of light passing through a sample. In either of these methods the quality of the data depends upon the minimum resolvable contrast of the light striking the camera. For this reason it is desirable to have a camera that can efficiently and accurately record low light levels with a high signal to noise ratio. In this article we present a method to modify control software for an inexpensive astronomy CCD camera to make it suitable for taking absorption images of cold atomic clouds. This system is capable of providing high-quality, 16-bit CCD absorption images of ultracold atomic rubidium clouds. The modified control software is also integrated into a Matlab graphical user interface (GUI), which also performs the necessary data analysis.

Absorption images are created when resonant, or near-

\footnotetext{
${ }^{a)}$ Electronic mail: dwhitaker@williams.edu

${ }^{b)}$ Present address: Department of Physics, Princeton University, Princeton, NJ 08544.
}

resonant, light is shone through an atomic sample. For light levels below saturation intensity, the transmitted intensity of a beam propagating along the $\hat{z}$-axis obeys Beer's law, $I$ $=I_{0} e^{-O D(x, y)}$, where $I_{0}$ is the intensity of light incident on the cloud and $O D(x, y)$ is the optical depth of the cloud as a function of position transverse to the beam. The optical depth depends upon both the column density of atoms through which the light passes and their photon scattering cross section, $\sigma_{0}$, as $O D=\sigma_{0} \int n(\vec{r}) d z$, where $n(\vec{r})$ is the density of the sample.

To take an absorption image of a cloud, three exposures are used. The first exposure $(A)$ is an image of the shadow cast by the cloud illuminated from behind by a short flash of resonant laser light. The second image $(B)$ is of an identical flash of light without a cloud. The third $(C)$ records the background light not coming from the flash by taking an identical exposure with no flash. These images are combined to give the $O D$ at each pixel, which is $O D=\log [(B-C) /(A-C)]$. In the regions where the absorption is greatest, image $A$ collects its minimum amount of light and has its lowest signal to noise ratio. The darkest resolvable feature, and consequently the dynamic range of the absorption image, depends on minimizing noise in the subtracted $A-C$ image.

Determining the minimally resolvable light level of the camera requires us to consider the sources of noise on each pixel. The three main sources of error in recording the number of photons striking a pixel are the shot noise associated with counting the number of photoelectrons, the readout noise, $N_{R}$, which depends on the electronics intrinsic to the camera, and the noise from the dark current, $N_{D}$. If one records a count of $N_{*}$ photons on a pixel, the associated noise level will be $N=\sqrt{N_{*}+N_{D}+N_{R}^{2}}$. ${ }^{12}$ Any portions of the image that collects fewer counts than the noise in the subtracted frame $A-C$ with not be readily resolved. For exposure times associated with absorption imaging $(t<1 \mathrm{~s})$ any cooled CCD will provide a sufficiently low dark noise that $N_{D}$ can be ignored. Therefore, the camera's performance depends on the readout noise and the magnitude of the signal.

To image clouds with large optical depths, one needs a 
camera with a low dark current and small readout noise. Since the absorption image of the cloud involves subtracting a dark frame to remove scattered light, the noise from images $A$ and $C$ must be added in quadrature, and even a pixel that receives no photons would have a noise of $\sqrt{2} N_{R}$. If we assume that the dark frame $C$ has no background light and $N_{*}$ is the value incident during image $A$, the total noise of the subtracted images is $\sqrt{N_{*}+2 N_{R}^{2}}$, meaning the signal to noise ratio can reach unity only at a level slightly above $\sqrt{2} N_{R}$, while usable images generally have about 10 times this value on the darkest pixels.

\section{IMAGING PROCEDURE}

To image our clouds we use an inexpensive camera ${ }^{13}$ designed for amateur astronomers, which has a low dark current and is sensitive at $780 \mathrm{~nm}$. The readout noise of the camera is $10 e$, which corresponds to 13 counts when taking 16-bit images, and the dark current is less than $1 e \mathrm{~s}^{-1}$. While these features are suitable for taking absorption images, the commercial control software included with the camera is not suitable for imaging cold clouds. The interline transfer CCD chip is not capable of reading out all of the rows in the camera simultaneously, but instead reads out the odd and even rows separately. The commercial software that is available with the camera compensates for this fact either by taking two identical exposures but reading out different rows each, or by wiping and re-exposing the second set of rows during the image so that the two rows have equal exposure times. While both of these methods are suitable for the long exposures used in astronomical imaging, multiple exposures of a single image cannot work on the sub-millisecond flashes used in absorption imaging.

We have modified the Visual Basic source code available with the camera to take a single exposure of the imaging flashes at full resolution. In our code the odd and even rows are read out sequentially after a single exposure. While the odd rows are read out, the even rows accumulate charge, but since an externally mounted shutter is closed during this time, only dark current accumulates. The readout time for each set of rows is $\sim 2 \mathrm{~s}$. In this period less than 1 electron of dark current accumulates on the even rows, which is substantially smaller than the readout error. Furthermore, the same additional dark current accumulates in the dark frame (image $C$ ), and is subtracted away in the final processed image.

The camera is controlled by a computer that is separate from the one used for critical timing in the experiment as described in Ref. 5. The two computers are connected through a serial port and a signal from the timing computer initiates the exposure process shortly before the atoms are ready to be imaged. Once the image has been recorded the camera software sends a signal to the control computer to repeat the process for images $B$ and $C$. The timing of our imaging procedure for a single shot is shown in Fig. 1. The timing of signals sent through the USB and serial ports is inconsistent enough that the exposure process must be initiated at least $250 \mathrm{~ms}$ before the image is taken. The shutter in front of the camera is opened $15 \mathrm{~ms}$ before the flash so that the shutter is completely open during the flash and the lock

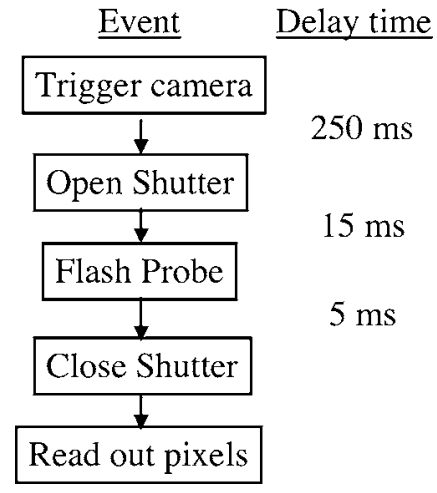

FIG. 1. A flowchart of the timing associated with taking a single image used for absorption imaging. The full exposure time of the camera is $700 \mathrm{~ms}$, but a mechanical shutter mounted on the front of the camera is closed during the vast majority of this time, over which essentially no noise from dark current accumulates.

of the probe laser is not affected by the vibrations of the shutter opening. Typical exposure times are $700 \mathrm{~ms}$, which are sufficiently long that flashes are consistently captured despite timing noise in the exposure process. The exposure time is short compared to the readout time of the camera, and even with exposures as long as $10 \mathrm{~s}$, there is no measurable effect from dark current noise accumulation during the exposure.

The program, which controls the exposure and reads the images from the camera, is a modified version of the Visual Basic code available with the camera. The program uses MSComm to send commands to the camera through the USB port, and has been compiled as an executable with its own GUI. This executable program ${ }^{14}$ allows for the user to control the exposure time of the camera for each image and writes the image data as a comma-delimited text file. The data can be output as either a single processed absorption image, $(A-C) /(B-C)$, or each image can be output separately. The images written to a file can be cropped to speed up the process of writing the data, but the entire CCD array must be read off in each frame. To take and record a single processed absorption image sequence takes approximately $20 \mathrm{~s}$, but this time can be reduced to about $12 \mathrm{~s}$ if the image is cropped.

We have also written the Visual Basic code from the imaging executable program so that it may be called by a Matlab GUI, which can also fit and manipulate the data from the images. In this case the Visual Basic routine is compiled into an ActiveX object and registered with the operating system so that Matlab can call it. Here, the data from the images are not written to a file, but are returned to Matlab as a onedimensional array that Matlab turns into a two-dimensional absorption image, which can be fitted to an appropriate distribution function as in Ref. 5. Furthermore, since data from the images are stored as a variable in Matlab, they can easily be manipulated with the command line interface of that program, allowing for easy real-time data analysis of images. With this program a thermal cloud can be imaged and fit in $\sim 20$ s. On our webpage ${ }^{14}$ we have included a copy of both the Visual Basic executable GUI and the Matlab version with instructions for their installation and operation. 

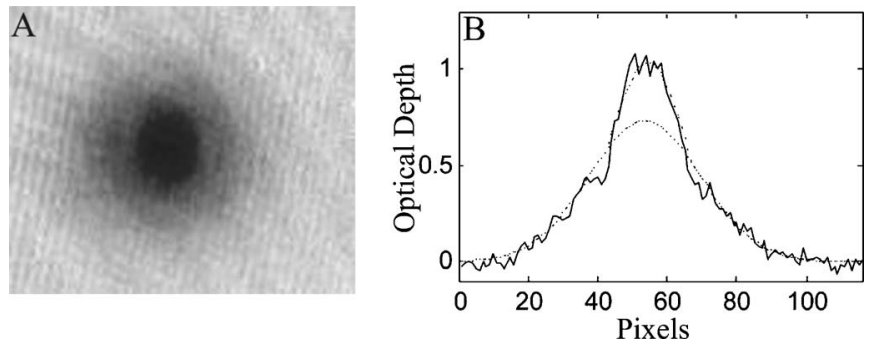

FIG. 2. (A) An absorption image of a Bose-condensed cloud of rubidium- 87 atoms with a substantial thermal component after being released from an optical trap. (B) a horizontal cut of the optical depth of image (A). The dotted curve is from a two-dimensional fit of the wings of the cloud to a Gaussian distribution. The dashed curve is from a two-component fit in which the condensate was fit to a Thomas-Fermi distribution. The sample shown has 20000 atoms in the condensate and 95000 in the thermal component at a temperature of $82 \mathrm{nK}$. The dominant source of the noise on this image comes from fringes associated with optical components in the imaging beam line.

\section{PERFORMANCE}

In our experiments clouds are illuminated with a $50 \mu \mathrm{s}$ flash of light from a $0.5 \mathrm{~mW}, 1 \mathrm{~cm}$ diameter beam. The flash duration was chosen to be a time that was short enough to prevent motional blurring of the clouds over our entire temperature range and to correspond to a full cycle of the modulation signal used to lock the probe laser to a spectral peak. These flashes give a signal of about 9000 counts/pixel in the normalization image, $B-C$. At these light levels the predominant noise is shot noise from counting the photons, or about 95 counts. As discussed above, the minimally resolvable $O D$ will depend on the noise level of the subtracted image, $A-C$. For a signal to be 10 times the combined readout noise of these two images it must have 180 counts, or a fractional transmission of 0.02 , which corresponds to $O D_{\max }=3.9$. We have observed clouds with $O D$ s exceeding 3 , but these images were noisy and flat in the darkest regions. Typically we let clouds expand until their $O D$ is less than 1.2 for the best data. Higher $O D$ s could be observed if we used longer flashes and increased the signal in our normalization image, but clouds with very high $O D$ s suffer from systematic errors such as lensing and do not provide accurate data even with a high signal to noise ratio.

An example of an image from a finite-temperature BEC is shown in Fig. 2(A), and a cross section of the cloud's $O D$ and fit to a bimodal velocity distribution ${ }^{10}$ is shown in Fig. 2(b). The maximum optical depth of the image shown is 1.0 and the primary source of noise is from fringes caused from etalon effects due to uncoated optics in the beam path. Condensates of fewer than 10000 atoms can easily be resolved from a thermal component with our system, and the camera is not the limiting factor on imaging noise. The quality of the images taken with this system is comparable to others using a front illuminated CCD such as in Ref. 5.

\section{DISCUSSION}

We have modified the control software of an inexpensive CCD camera designed for amateur astronomers to take highquality 16-bit absorption images of ultracold rubidium-87 atom clouds. Our system can readily resolve small condensate fractions from a thermal cloud and has noise levels that are insignificant compared to those from the optical system used to take the pictures. While we have focused our discussion here on using this camera for absorption images, it can also be used to perform fluorescence or phase contrast imaging $^{11}$ on clouds. Either method requires a camera capable of recording short light flashes with a low signal to noise and high sensitivity, which we have demonstrated.

\section{ACKNOWLEDGMENTS}

The authors would like to thank Steve Souza for helpful discussions about imaging with CCDs and Eric Cornell for his useful comments. The authors are grateful to Terry Platt for technical assistance in modifying the original source code from Starlight Xpress. The authors also wish to thank Jonathan Morgan-Leaman, Sarah Iams, and Brian Munroe for their help in writing the Visual Basic code.

${ }^{1}$ A. A. Arnold, J. S. Wilson, and M. G. Boshier, Rev. Sci. Instrum. 69, 1236 (1998)

${ }^{2}$ L. Ricci, M. Wiedemüller, T. Esslinger, A. Hemmerich, C. Zimmermann, V. Vuletic, W. König, and T. W. Hänsch, Optics Commun. 117, 541 (1995).

${ }^{3}$ K. B. MacAdam, A. Steinbach, and C. Wieman, Am. J. Phys. 60, 1098 (1992).

${ }^{4}$ C. Wieman, G. Flowers, and S. Gilbert, Am. J. Phys. 63, 317 (1995).

${ }^{5}$ H. J. Lewandowski, D. M. Harber, D. L. Whitaker, and E. A. Cornell, J. Low Temp. Phys. 132, 309 (2003).

${ }^{6}$ W. Hänsel, J. Reichel, P. Hommelhoff, and T. W. Hänsch, Phys. Rev. A 64, 063607 (2001).

${ }^{7}$ M. Barrett, J. Sauer, and M. Chapman, Phys. Rev. Lett. 87, 010404 (2001).

${ }^{8}$ T. Kinoshita, T. R. Wenger, and D. S. Weiss, Phys. Rev. A 71, 011602(R) (2005).

${ }^{9}$ P. Ahmadi, B. P. Timmons, and G. S. Summy, Phys. Rev. A 72, 023411 (2005).

${ }^{10}$ W. Ketterle, D. S. Durfee, and D. M. Stamper-Kurn, in Proceedings of the International School of Physics-Enrico Fermi, edited by M. Inguscio, S. Stringari, and C. E. Wieman (IOS Press, Amsterdam, 1999), p. 67.

${ }^{11}$ M. Andrews, M.-O. Mewes, N. van Druten, D. Durfee, D. Kurn, and W. Ketterle, Science 273, 84 (1996).

${ }^{12}$ S. B. Howell, Handbook of CCD Astronomy (Cambridge University Press, Cambridge, 2000).

${ }^{13}$ MX716 camera from Starlight Xpress Ltd., Berks, England.

${ }^{14}$ Both the executable GUI to take and save images and the Matlab GUI, which incorporates fitting routines, as well as their source codes and instructions are available for download at www.williams.edu/Physics/ dwhitaker/imaging/. 